
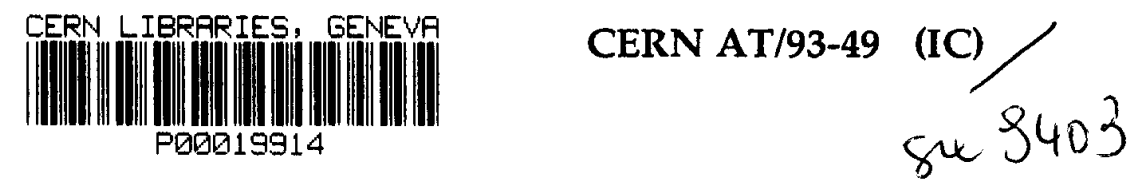

\title{
Laboratory use of industrial control systems
}

A. Rijllart, L. Avot, D. Brahy, D. Jégou and R. Saban

\begin{abstract}
Industrial control system manufacturers supply the building blocks for the control of industrial equipment or specific process control applications. Although the laboratory environment is different in many aspects (prototyping, evolution, frequent reconfiguration), the usage of these building blocks remain attractive because of their general purpose nature, their cost and the large spectrum of available types. In this paper we present three projects which have been implemented using both industrial control system building blocks (PLCs, controllers, digital and analogue plug-in I/O cards) and commercial software packages (LabView, VisualBasic) for the man-machine interface, the data acquisition and archiving, and the process control. This approach has proved to be economical, easy and fast to implement.
\end{abstract}

ICALEPCS'93 - International Conference on Accelerator and Large Experimental Physics Control Systems, Berlin, Germany, October 18-22, 1993 


\section{Introduction}

The Industrial Controls group in the Accelerator Technology Division at CERN investigates the possibilities of implementing industrial control systems in areas where up to now application specific control systems have been used [1]. Laboratory installations are typical examples where such systems are being used because of their special character of being unique and evolutionary. With the availability of a wide range of industrial control system building blocks at low cost and commercial software packages, industrial control can successfully be implemented in the laboratory environment. Three examples are described below.

The first is a superconducting accelerating cavity rinsing installation where cavities are being cleaned before use. The rinsing is done with ultra-pure water (18 $M \Omega$ ) pressurized up to 100 bar followed by a rinsing with ethanol. The idea was to fully automate the rinsing procedure and to safeguard the ultra-pure water from any contamination.

The second is the automation of a cryogenic test installation for the superconducting cavities. In this installation the liquid helium transfer to and from two cryostats had to be controlled, together with the recovery of the helium gas produced in these operations. Procedures specific to cavity testing, such as pumping the helium bath down to 10 mbar pressure and re-pressurizing are part of the automation.

The third is a three-axis robot arm which carries an analytic head for the inner surface inspection of the superconducting cavities once a defect has been located in the cryogenic test installation. The analytic head has to be positioned with a precision of $0.5 \mathrm{~mm}$ at a distance of $6.5 \mathrm{~mm}$ from the surface. Movements along the surface plane or perpendicular to it must be possible. Special care has been taken to minimize the chance of a collision between head and cavity wall and to limit the damage in the event of a collision.

\section{A LabVIEW and PLC based system for the rinsing of the LEP200 cavities}

The cavity rinsing installation (fig. 1) consists of a ultra-pure water storage tank, a variable flow pump, a recirculation pump and an ethanol storage tank. The ultra-pure water tank gets filled with pre-purified water which is filtered once more and recirculated regularly. The ethanol tank gets filled with an external tank. The rinsing procedure of a cavity includes usually rinsing with ultra-pure water, followed by rinsing with ethanol to take away the water and to speed-up the 
drying. Exceptionally a cavity gets rinsed with pre-purified water instead of the ultra-pure water. As the ultra-pure water and the ethanol pass through the same pump and lines there is a physical connection between the two circuits. A strict separation, however, has to be maintained at the level of the ultra-pure water storage tank. The ultra-pure water circuit once contaminated with ethanol or even pre-purified water will need a sterilization before becoming ultra-pure again, which is a tedious operation.

To obtain the functionality and robustness necessary for the control and contamination protection of the ultra-pure water rinsing installation a PLC has been chosen. For the functions of supervisor and operator interface a Macintosh has been chosen with the LabVIEW software which communicates over a serial link with the PLC using the $3964 \mathrm{R}$ protocol.

The PLC has been programmed using Grafcet and can be operated in its simplest form by means of a few push buttons to command the most frequently used rinsing cycle. The LabVIEW application provides an animated synoptic diagram of the installation with a history file and full operation facilities with buttons on the screen. The parameters of the rinsing cycles in the PLC can be modified and down-loaded from the Macintosh. A fully manual debug mode has been provided as well under LabVIEW where the operator has full control over the installation with a risk of contaminating the ultra-pure water circuit. Means of access to this manual mode and to parameter modification are protected with a password.

\section{A PLC based control system for a cryogenic test facility of the LEP200 cavities}

The helium cryogenic test facility (fig. 2) consists of two cryostats, a pump, a liquefier with a dewar, a storage dewar and a gas recovery system. The cryostats are filled from the storage dewar, which in turn is filled from the liquefier dewar when necessary. After filling the cryostats, the helium bath usually has to be pumped down to 10 mbar (corresponding to a temperature of $2.5 \mathrm{~K}$ ) and repressurized before a specific cavity defect location measurement takes place. During cavity processing, an operation of putting at least $100 \mathrm{~W}$ of RF power in the cavity for 24 hours, the level in the cryostat has to be kept between $75 \%$ and $90 \%$ of the maximum. At the end of a test the cryostat has to be emptied and the cavity and cryostat are warmed up to room temperature. 
All transfer of liquid helium produces helium gas. This gas is returned to the recovery system which has a fixed maximum capacity. When there is a risk of exceeding the maximum capacity procedures producing large quantities of gas have to be suspended.

We have chosen a Makmodul PLC as a stand-alone control system for this installation. The PLC met the requirements of being programmable in a high level language and have the possibility to use a colour screen for the display of animated synoptic diagrams, time histograms and multiple windows for easy operator interaction. The programming of the PLC has been done in Forth [2] using the multi-tasking facilities where control functions, displays and operator interaction can run simultaneously.

\section{A PC based system for an inner surface analysis of the LEP200 cavities}

A commercial analytical measurement instrument has been mounted on a CERN built robot arm for inner surface inspection of the LEP200 cavities in vertical position (fig. 3). This instrument performs scanning Auger and secondary electron microscope measurements. It needs to be operated in a vacuum of better than $10^{-7}$ mbar and at a distance of $6.5 \pm 1 \mathrm{~mm}$ from the surface. The robot arm, which has one articulation, needs three axis movements, vertical $(Z)$, inclination (a) and rotation ( $f$ ) to reach any position in the top halves of the 4-cell cavity. To reach the other halves the cavity will have to be reversed.

Defect location measurements during cryogenic testing of a cavity have an accuracy of $\pm 10 \mathrm{~mm}$. The scanning Auger measurement covers an area of $0.5 \times 0.5$ $\mathrm{mm}$, so some searching with small head displacement is required. For the robot control system we have chosen a three-axis stepping motor solution with a PC plug-in controller board. The controller operates with string type commands and to provide a user-friendly interface with position visualisation and easy move commands a VisualBasic program was written.

To avoid collisions between the head and the cavity wall, absolute position encoders have been used and redundancy has been added for the most critical movement (a) in the form of an electrolytic tilt sensor. To reduce damage in the event of a collision and to take into account all the other conditions (vacuum, valve positions, etc.) needed to be able to operate, a separate PLC based interlock system has been implemented which works in parallel with the motor controller and can stop all movements in case of a serious problem. 


\section{Conclusions}

Industrial control system building blocks can successfully be used for laboratory use as shown in the three examples. Care has to be taken in the design of the system to allow flexibility and extensions. Special adaptations are often needed to obtain satisfactory performance, but the concept of the industrial approach remains valid because of the large variety of available building blocks which result in short implementation times and low cost.

\section{References}

[1] M. Rabany, Proceedings of ICALEPS '91, Tsukuba, Japan (1991) 269.

[2] P. Courtois, Le concept Forth, Editests (1983). 
Figure captions

Fig. 1 Schematic structure of cavity rinsing project

Fig. 2 Schematic diagram of cryogenic test installation

Fig. 3 Schematic diagram of the three-axis robot control system 
Fig. 1

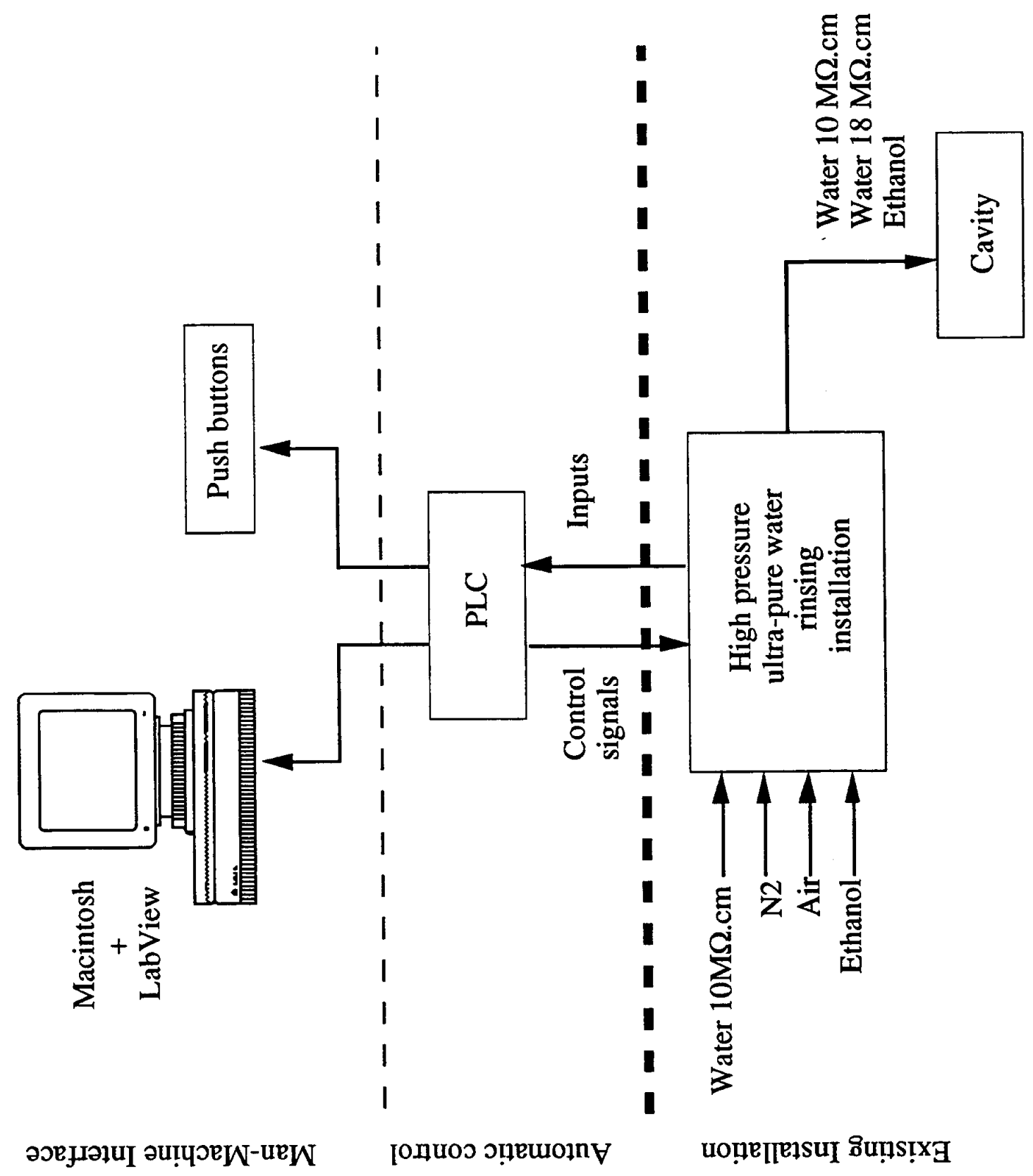




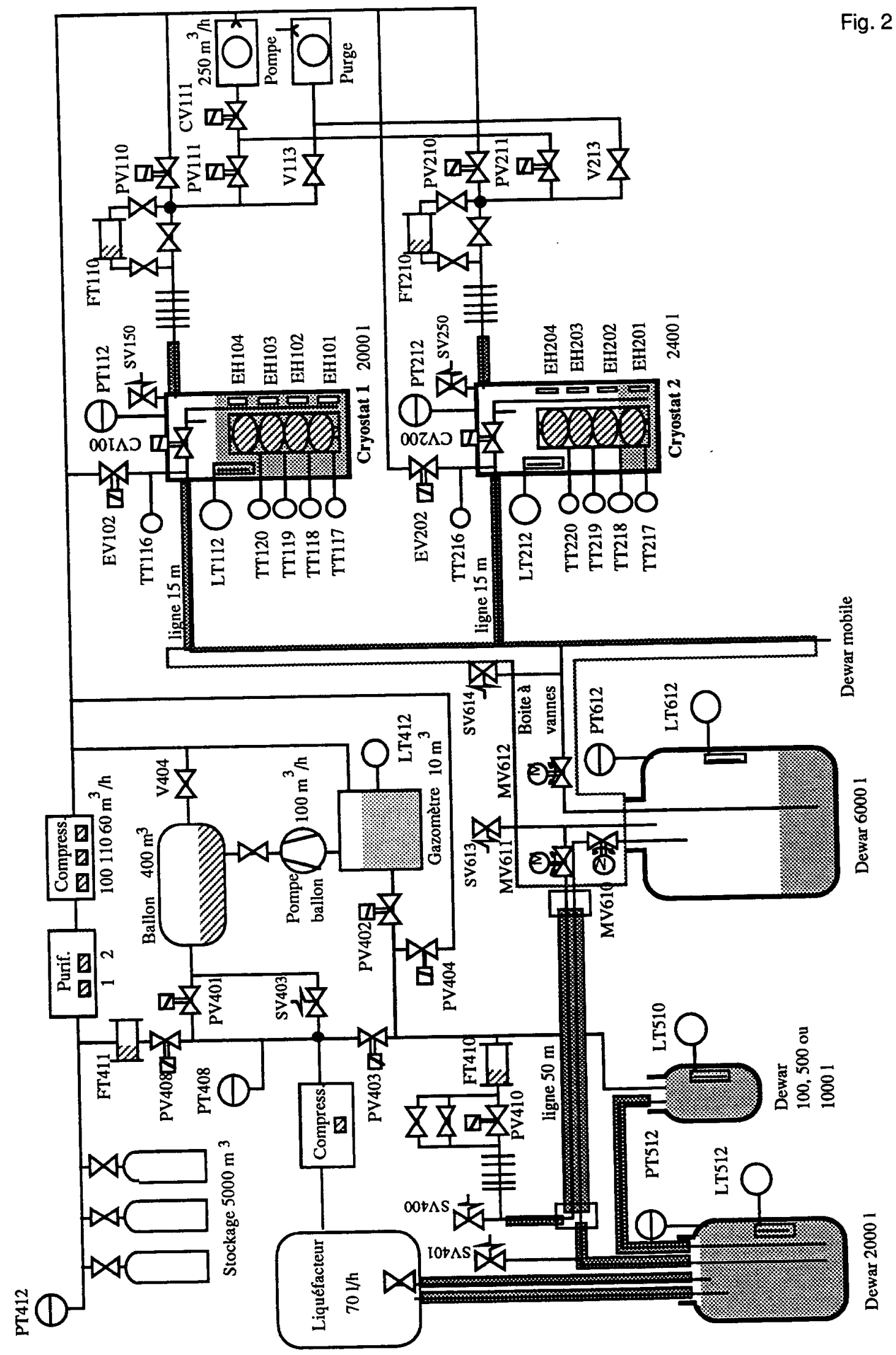




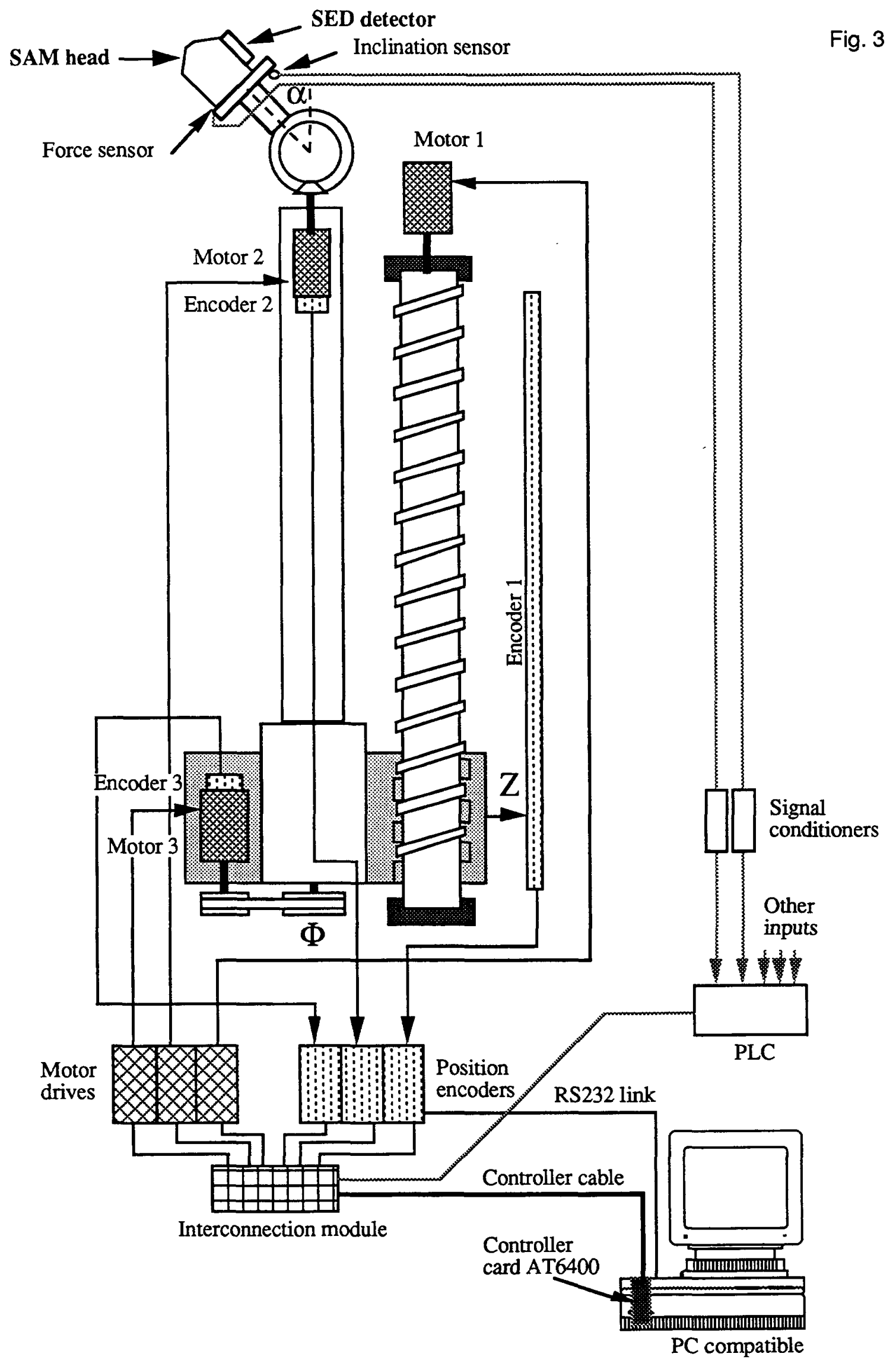


